\title{
Induction of lipid oxidation by polyunsaturated fatty acids of marine origin in small intestine of mice fed a high-fat diet
} Evert M van Schothorst*1, Pavel Flachs ${ }^{2}$, Nicole LW Franssen-van Hal ${ }^{1}$, Ondrej Kuda ${ }^{2}$, Annelies Bunschoten ${ }^{1}$, Jos Molthoff ${ }^{1}$, Carolien Vink ${ }^{1}$, Guido JEJ Hooiveld ${ }^{3}$, Jan Kopecky² and Jaap Keijer ${ }^{1,3,4}$

\author{
Address: ${ }^{1}$ Food Bioactives group, RIKILT - Institute of Food Safety, Wageningen UR, PO Box 230, 6700 AE Wageningen, the Netherlands, \\ ${ }^{2}$ Department of Adipose Tissue Biology, Institute of Physiology, Academy of Sciences of the Czech Republic, Prague, Czech Republic, ${ }^{3}$ Nutrition, \\ Metabolism and Genomics Group, Division of Human Nutrition, Wageningen University, Wageningen, the Netherlands and ${ }^{4}$ Human and Animal \\ Physiology, Wageningen University, Wageningen, the Netherlands \\ Email: Evert M van Schothorst* - evert.vanschothorst@wur.nl; Pavel Flachs - flachs@biomed.cas.cz; Nicole LW Franssen-van \\ Hal - N.Franssen@flexgen.nl; Ondrej Kuda - kuda@biomed.cas.cz; Annelies Bunschoten - annelies.bunschoten@wur.nl; \\ Jos Molthoff - jos.molthoff@wur.nl; Carolien Vink - Carolien.Vink@nizo.nl; Guido JEJ Hooiveld - guido.hooiveld@wur.nl; \\ Jan Kopecky - kopecky@biomed.cas.cz; Jaap Keijer - jaap.keijer@wur.nl \\ * Corresponding author
}

Published: 16 March 2009

BMC Genomics 2009, 10:110 doi:10.1186/1471-2164-10-110
Received: 18 December 2008

Accepted: 16 March 2009

This article is available from: http://www.biomedcentral.com/147/-2/64/10/110

(C) 2009 van Schothorst et al; licensee BioMed Central Ltd.

This is an Open Access article distributed under the terms of the Creative Commons Attribution License (http://creativecommons.org/licenses/by/2.0), which permits unrestricted use, distribution, and reproduction in any medium, provided the original work is properly cited.

\begin{abstract}
Background: Dietary polyunsaturated fatty acids (PUFA), in particular the long chain marine fatty acids docosahexaenoic (DHA) and eicosapentaenoic (EPA), are linked to many health benefits in humans and in animal models. Little is known of the molecular response to DHA and EPA of the small intestine, and the potential contribution of this organ to the beneficial effects of these fatty acids. Here, we assessed gene expression changes induced by DHA and EPA in the wildtype C57BL/ 6] murine small intestine using whole genome microarrays and functionally characterized the most prominent biological process.
\end{abstract}

Results: The main biological process affected based on gene expression analysis was lipid metabolism. Fatty acid uptake, peroxisomal and mitochondrial beta-oxidation, and omegaoxidation of fatty acids were all increased. Quantitative real time PCR, and -in a second animal experiment- intestinal fatty acid oxidation measurements confirmed significant gene expression differences and showed in a dose-dependent manner significant changes at biological functional level. Furthermore, no major changes in the expression of lipid metabolism genes were observed in the colon.

Conclusion: We show that marine $\mathrm{n}-3$ fatty acids regulate small intestinal gene expression and increase fatty acid oxidation. Since this organ contributes significantly to whole organism energy use, this effect on the small intestine may well contribute to the beneficial physiological effects of marine PUFAs under conditions that will normally lead to development of obesity, insulin resistance and diabetes. 


\section{Background}

Diets rich in polyunsaturated fatty acids (PUFA) of $n-3$ series show many beneficial health effects, both in animal models and humans. These include effects on cardiovascular and immune systems, on glucose homeostasis, as well as on the accumulation of body fat (e.g. reviewed by [1-3]). However, recent epidemiological studies started a debate on the possible health benefits of n-3 PUFA $[4,5]$. To resolve the potential health benefits of these fatty acids, knowledge of the underlying mechanisms is needed.

To elucidate molecular effects of n-3 PUFA in vivo, gene expression analyses have been undertaken in animal models using a variety of dietary fatty acids in several tissues, including brain, liver, heart, and adipose [6-16]. The majority of those studies focused on liver and white adipose tissue (WAT), which is not surprising given the fact that these are considered the main target organs in a dietary intervention with fatty acids. Since the intestine contributes to a significant extend to the resting metabolic rate and daily energy expenditure [17], it is of relevance to also understand the effects on this organ. Recent studies $[18,19]$ also showed a clear and significant difference of intestinal gene expression between diets high in diacylglycerol versus triacylglycerol, indicating a profound contribution of the small intestine to fatty acid metabolism. Moreover, induction of lipid catabolism genes in the intestine may be involved in the anti-obesity effect of diacylglycerols as compared with triacylglycerols $[18,19]$ and it may even contribute to a differential sensitivity of two inbred mice strains to an obesogenic high-fat diet [20].

Since the most prominent health benefits have been associated with the long-chain n-3 PUFA of marine origin (for references see $[21,22])$, we have investigated the molecular effects of eicosapentaenoic acid (EPA; 20:5 n-3) and docosahexaenoic acid (DHA; 22:6 n-3) in n-3 high-fat diets. These diets, which do not differ in the total amount of fat relative to control, will be further referred to as EPA\&DHA. In our previous studies using similar diets, we showed an anti-adipogenic effect of EPA\&DHA $[8,23]$, which was associated with induction of mitochondrial biogenesis and beta-oxidation of fatty acids in WAT, but not in the liver [8].

We hypothesized that, using long-term dietary intervention studies, dietary fatty acid composition may modulate gene expression and lipid metabolism in the intestine, and that especially EPA and DHA may stimulate expression of genes involved in lipid catabolism. To examine this, we performed gene expression analysis of the mouse small intestine and colon, using whole genome oligonucleotide arrays and validation experiments using quantitative real time PCR (qRT-PCR). Results were confirmed in an additional animal experiment by both qRT-PCR and functional intestinal fatty acid beta oxidation measurements.

\section{Results}

\section{Phenotypic effects of EPA\&DHA}

Dietary intervention with EPA\&DHA in wildtype mice resulted in anti-adipogenic and anti-diabetic effects as described before: significantly lower body weight and epididymal adipose tissue weight, while food intake was non-significantly different $[8,23]$. Furthermore, the intake of EPA\&DHA increased adiponectin expression and secretion from WAT, and protected the mice against induction of insulin resistance by the high-fat diet [23]. Indeed, glucose tolerance tests showed significantly increased glucose tolerance (decreased area under the curves) by increasing amounts of EPA\&DHA in the diets, correlating with decreased fasting plasma insulin levels (data not shown). This was associated with induction of mitochondrial biogenesis and beta-oxidation of fatty acids in WAT based on gene and protein expression, but not in the liver [8]. However, the role of the intestine in the improved whole-body metabolic phenotype has not yet been analysed in detail.

\section{Effects of EPA\&DHA on small intestinal gene expression; analysis of genes expressed in both control and intervention group}

To investigate the effects of EPA\&DHA on gene expression in the intestinal tract, we isolated RNA from scrapings from small intestine of mice following a 4-week dietary intervention. We compared, using whole genome microarray analysis as initial step, the control sHF diet, which was rich in ALA and free of EPA or DHA, with the isocaloric sHFf-F2 diet, in which $44 \%$ of lipids were replaced by an EPA and DHA concentrate.

In total, 155 probesets, corresponding to 134 well-annotated genes, showed a significant regulation with an absolute fold change $(\mathrm{FC}) \geq 2.0$ [see Additional file 1]. This list of 134 genes comprised 110 unique genes, and increased expression was observed for 80 (102 probesets) unique genes, while 30 (32 probesets) unique genes showed decreased expression (Table 1). Functional interpretation using gene ontology and pathway analysis showed metabolism as highly regulated, which included changes in fatty acid uptake, fatty acid oxidation and cholesterol biosynthesis, amongst others. Of note, most, if not all, pathways selected were linked together by the energy molecule acetyl-CoA. The approach using a FC $\geq 1.5$ as threshold resulted in a similar list of regulated pathways, of which metabolism, and especially lipid metabolism ranked highest (a more detailed description of these approaches and detailed results are described additionally [see Additional file 2]). Since it is known that a dietary intervention at physiological levels generally gives small effects on gene expression changes $([24,25]$, own unpublished observa- 
Table I: Differentially expressed, unique genes classified in biological processes.

\begin{tabular}{|c|c|c|c|}
\hline Process & Upregulated & Downregulated & Total \\
\hline Metabolism & 40 & 9 & 49 \\
\hline Transport & 4 & 3 & 7 \\
\hline Apoptosis/cell cycle & 4 & 2 & 6 \\
\hline Cell adhesion/homeostasis/structure & 4 & I & 5 \\
\hline Digestion & 4 & 0 & 4 \\
\hline Immune system & i & 3 & 4 \\
\hline Hemopoiesis & I & 2 & 3 \\
\hline Transcription/translation & 3 & 0 & 3 \\
\hline Signal transduction & 2 & 0 & 2 \\
\hline Steroid hormone metabolism & 2 & 0 & 2 \\
\hline Miscellaneous/unknown & 8 & 7 & 15 \\
\hline & 73 & 27 & 100 \\
\hline
\end{tabular}

Unique genes (expressed in both dietary groups, $\mathrm{FC} \geq 2.0$ ) are classified in II processes, and ranked on total number of genes per process. Number of genes upregulated or downregulated by EPA\&DHA in small intestine is as indicated.

tions), and the sufficiently large group of genes with $\mathrm{FC} \geq$ 2.0, we focussed on those 100 unique, differentially expressed genes (see Table 1).

Detailed inspection of the expression data ([see Additional file 1]; see also for full names of the genes) revealed that EPA\&DHA induced expression of the genes involved in branched chain and/or straight chain fatty acid $\beta$-oxidation occurring in peroxisomes and mitochondria, and fatty acid $\omega$-oxidation, the latter is indicated by the increased expression of Cyp4a10 $(\mathrm{FC}=5.6)$. Furthermore, induction of the peroxisomal biogenesis factor gene Pex11a $(F C=2.2)$ might imply an increase in the number of peroxisomes.

In mitochondria, $\beta$-oxidation downstream of peroxisomal branched chain oxidation was upregulated by EPA\&DHA, as suggested by increased expression of the genes CPT1a $(\mathrm{FC}=2.9)$, and Hadhb $(\mathrm{FC}=2.2)$ amongst others. Activity of carnitine palmitoyltransferase 1 (encoded by Cpt1a) is rate-limiting in mitochondrial fatty acid uptake for $\beta$-oxidation. The gene encoding 3hydroxy-3-methyglutaryl-CoA synthase (Hmgcs2) was also very strongly upregulated ( $\mathrm{FC}=4.6)$, in accordance with the fact that in liver this enzyme is the rate limiting enzyme in the synthesis of ketone bodies from the acetylCoA generated by fatty acid $\beta$-oxidation. Importantly, also expression of Pdk4 was increased by EPA\&DHA (FC = 3.0), which strongly suggested a switch from glycolysis to fatty acid oxidation [26]. Many of the aforementioned genes are targets of peroxisome proliferator activated receptor $(P P A R)$ alpha $[27,28]$, which itself was also upregulated by EPA\&DHA $(\mathrm{FC}=2.0)$.

Cholesterol uptake from the lumen into intestinal tissue was increased as observed by increased gene expression of
Cd36 (FC = 2.3) and Scarb1 $(\mathrm{FC}=2.2)$ [29]. Simultaneously, of the 21 genes included in the cholesterol biosynthesis pathway from acetyl-CoA to cholesterol, 2 were not expressed, while the majority showed an inhibition by EPA\&DHA, including the rate-limiting enzyme squalene epoxidase (Sqle, FC $=-2.1$ ). Clearly, such a cooperative inhibition of the majority of the genes in this pathway suggests an orchestrated function within the small intestine. However, the main transcription factor regulating this pathway, Srebf1 (SREBP), did not show differential expression. Regulation by n-3 PUFAs was likely given the fact that we could not detect a difference in the cholesterol content between the two diets (data not shown), but the exact mechanisms involved remain unexplained. Finally, downstream steroid hormone biosynthesis was upregulated as shown by a few family members of $H s d 3 b$ $(H s d 3 b 2: \mathrm{FC}=2.3 ; H s d 3 b 3 \mathrm{FC}=2.0)$ and $H s d 17 b(H s d 17 b 4$ two probesets: $\mathrm{FC}=1.6$ and 2.0; Hsd17b13 FC $=2.4$ ).

\section{Transcription factor identification by promoter analysis}

Literature data and annotation analysis was used to filter the set of regulated genes (see Methods). From the resulting 50 unique genes the promoter region was retrieved, which was used for enrichment analysis of transcription factor binding sites (TFBS). This resulted in a network of 47 genes and linked transcription factors (TFs) that comprised 42 unique input genes, including two TFs (PPARalpha and Dbp), and another 5 non-regulated TFs (NF- $\kappa \mathrm{B}$, Stat3, Sp1, Ahr, and Arnt1; [see Additional file 3]). The majority of differentially regulated genes contained binding sites for two transcription factors: PPARalpha (27 genes), which itself was significantly differentially regulated (see above), and NF-кB (20 genes). These data are in line with the well-known capacity of fatty acids to activate PPARalpha [30], as well as their known effects on NF- $\kappa B$ and Stat3 $[3,31]$. 
Effects of EPA\&DHA on small intestinal gene expression; analysis of genes expressed exclusively in either control or intervention group

In addition to the genes that showed expression above the threshold in both groups, we also analysed the 159 probesets being expressed in only one diet group and showing differential expression. We focused on those genes showing a FC $\geq 2$.0. In this way, fidelity of the dataset is within acceptable limits as most of these genes are expressed at low abundance. This resulted in 23 unique genes expressed only in the mice fed the EPA\&DHA diet, while 47 unique genes showed only expression in the control group. All genes were classified into biological processes (Table 2, [see Additional file 4]), which largely overlapped with the pathways identified using the first subset. Of note, metabolism was also ranked highest, among which are the lipid metabolism genes acetyl-Coenzyme A carboxylase- $\beta$ (Acacb), Cpa2, and the peroxisomal protein encoded by $A \cot 3$, which all showed an induction from being absent in the control group to clear expression in EPA\&DHA dietary group.

\section{Inter-individual variation analysis and confirmation by real time $q R T-P C R$}

Confirmation of differentially expressed genes was first performed using qRT-PCR. We selected a large panel of target genes covering multiple pathways and absolute FC values ranging from 1.3 to $>4$. Expression changes (up- or downregulation) were confirmed for all genes (Table 3) and the FC was quite similar in all cases when compared to the microarray data.

To investigate in more detail inter-individual variation in gene expression and significance of the induced gene expression changes by nutritional treatment, we selected the following 6 genes representing the major pathways being influenced by the diet intervention (see above): fatty acid $\beta$-oxidation in peroxisomes (Acaa1a) and mito- chondria (Cpt1a and Acacb), the switch between glycolysis and fatty acid oxidation (Pdk4), biosynthesis of steroid hormones $(H s d 3 b)$, and biosynthesis of cholesterol (Sqle). For all genes we observed that the mean gene expression ratios ( $\mathrm{n}=10-11$ per dietary group) were similar to the observed ratios by microarray analysis of pooled samples. More importantly, gene expression changes using individual samples were statistically significant for all genes (Figure 1, Table 3).

\section{Colon}

The genes Acaa1a, Cpt1a, and Sqle, representing three pathways differentially regulated in small intestine, were analyzed by qRT-PCR in individual colon samples $(\mathrm{n}=$ 10). In none of the cases a significant difference in gene expression was observed (Figure 2). Exclusion of the two animals from the control group which showed lowest expression for these three genes (10-fold or more) did not influence the outcome of this analysis (maximum $\mathrm{FC}<$ 1.15, all non-significant; data not shown). This underscores the different function of the small intestine (mainly uptake and transfer of nutrients) and the colon (mainly water absorption).

\section{Biological and physiological validation in $2^{\text {nd }}$ animal experiment}

To further validate the observed significant gene expression changes, we performed an independent second animal experiment and analysed small intestinal gene expression by qRT-PCR. Moreover, the increased gene expression suggesting increased intestinal fatty acid beta oxidation was functionally analysed, and finally, we used two EPA\&DHA diets in order to investigate a doseresponse relationship. In this experiment, cHF-based diets were used. Although the micronutrient composition of the control cHF diet is not defined as precisely as the semisynthetic sHFf diet used in the first experiment, and it is based on (n-6 PUFA rich) corn oil rather than on (ALA-

Table 2: Differentially expressed genes with exclusive expression in only EPA\&DHA or control dietary group.

\begin{tabular}{|c|c|c|c|}
\hline Process & Expressed in EPA \& DHA diet & Expressed in control diet & Total \\
\hline Metabolism & 11 & 4 & 15 \\
\hline Transcription/translation/splicing & 3 & 6 & 9 \\
\hline Cell adhesion/proliferation/differentiation/structure & - & 7 & 7 \\
\hline Signal transduction & - & 7 & 7 \\
\hline Transport & I & 5 & 6 \\
\hline Protein folding/modification & i & 4 & 5 \\
\hline Cell cycle & I & I & 2 \\
\hline Growth factor & 1 & 1 & 2 \\
\hline Miscellaneous/unknown & 5 & 12 & 17 \\
\hline & 23 & 47 & 70 \\
\hline
\end{tabular}

Annotated, unique genes ( $F C \geq 2$ ) were classified in biological processes. Processes were ranked on total number of genes being differentially expressed in small intestine. 
Table 3: Microarray data validation by qRT-PCR

\begin{tabular}{|c|c|c|c|c|}
\hline \multirow[b]{2}{*}{ Genes } & \multirow[b]{2}{*}{ Probeset ID } & \multirow{2}{*}{$\begin{array}{c}\text { Microarray ratio } \\
\text { Pool }\end{array}$} & \multicolumn{2}{|r|}{ qRT-PCR ratio } \\
\hline & & & Pool & mean ratio $(n=10-1 \mid)$ \\
\hline Acaala & |4560|I_x_at & 1.67 & 2.00 & $2.52 *$ \\
\hline Acaalb & |42445|_at & 2.19 & 1.75 & \\
\hline \multirow[t]{2}{*}{ Acaa la/Acaa lb } & 1416946_a_at & 1.87 & & \\
\hline & 1416947_s_at & 1.59 & & \\
\hline Acacb & 1427052_at & $6.9 \mathrm{a}$ & n.d.c & $3.91 *$ \\
\hline \multirow[t]{2}{*}{ Acoxl } & 1416409_at & 1.24 & 1.34 & \\
\hline & 1416408_at & 1.35 & & \\
\hline Acox2 & |420673_a_at & 2.91 & 1.97 & \\
\hline Acox3 & 1420684_at & n.c. ${ }^{b}$ & -1.23 & \\
\hline \multirow[t]{2}{*}{ Cptla } & 1434866_x_at & 2.87 & 1.87 & $2.79 * *$ \\
\hline & 1438156_x_at & 2.60 & & \\
\hline Cpt2 & 1416772_at & 1.39 & 1.44 & \\
\hline Ela2 & |44828I_a_at & 2.04 & 4.26 & \\
\hline \multirow[t]{5}{*}{ Ela3b } & 1415884_at & 2,64 & 7.89 & \\
\hline & |4356||__x_at & 4,20 & & \\
\hline & 1415883_a_at & 4,38 & & \\
\hline & 1437326_x_at & 4,72 & & \\
\hline & 1435012_x_at & 5,17 & & \\
\hline H2-Q10 & |425|37_a_at & -8.22 & -2.39 & \\
\hline \multirow[t]{2}{*}{ HmgcsI } & 1433443_a_at & -1.82 & -2.00 & \\
\hline & 1433446_at & -1.57 & & \\
\hline \multirow[t]{2}{*}{ Hmgcs2 } & |43|833_a_at & 3.61 & 3.97 & \\
\hline & 1423858_a_at & 4.56 & & \\
\hline $\mathrm{Hsd} 3 \mathrm{~b} 2 / 6$ & 1460232_s_at & 2.50 & n.d. & $1.91 *$ \\
\hline Hsd3b3 & |427377_x_at & 2.00 & & \\
\hline \multirow[t]{2}{*}{ Modl } & 1416632_at & 2.16 & 2.60 & \\
\hline & |430307_a_at & 2.13 & & \\
\hline \multirow[t]{2}{*}{ Pckl } & 1423439_at & 2.17 & 2.21 & \\
\hline & |439617_s_at & 2.46 & & \\
\hline Pdk4 & |417273_at & 3.01 & n.d. & $3.24 *$ \\
\hline Sqle & 1415993_at & -2.14 & -2.35 & $-1.55 *$ \\
\hline
\end{tabular}

Genes are sorted alphabetically. In several cases gene family members were included for validation, although they did not show differential microarray gene expression. Data is represented by ratio of EPA\&DHA/control diet. For qRT-PCR, data shown is obtained using a pool of samples (pool), and/or individual samples (EPA/DHA: $n=11$, control diet: $n=10$ ) and the mean ratio was calculated. a this probeset is non-expressed (absent) in control diet; ratio is 6.9 ; b ${ }^{n}$.c.: not changed. ${ }^{c}$ n.d.: not analyzed; statistical significance was calculated using Student's $t$-test: ${ }^{*} p<0.05$, ${ }^{* *}$ $\mathrm{p}<0.01$.

rich) flaxseed, the control cHF diet promotes accumulation of fat much better compared with the sHFf diet and hence it allows for the detection of the anti-obesity effect of n-3 PUFA [22]. In addition to the control cHF diet, also two other cHF-based diets with 15 and $44 \%$ of its lipids replaced by EPA\&DHA (cHF-F1 and cHF-F2, respectively) were used. Gene expression analysis of genes involved in mitochondrial beta oxidation (Cpt1a and Acacb) independently and significantly confirmed the observed significant differences (Figure 3a,b). Moreover, these results showed a significant dose-response effect: increased gene expression upon increased EPA\&DHA content in the diets (Figure 3a,b). Furthermore, functional analysis indeed showed a significant dose-dependent increase in intestinal fatty acid beta oxidation upon increased EPA\&DHA content in the diet compared to control diet: lowest levels in the control group, intermediate increased levels in the
cHF-F1 group and highest levels in the cHF-F2 group (Figure 3c).

\section{Discussion}

Dietary intake of EPA\&DHA induced changes in gene expression in the small intestine, including many metabolic genes. Especially genes involved in lipid catabolism were upregulated. In contrast, a large cluster of the genes engaged in cholesterol biosynthesis was downregulated. Importantly, all these effects were specifically induced by long-chain n-3 PUFA, EPA and DHA, as compared with their precursor ALA, and could not be detected in the colon for a selected set of genes.

It is tempting to suggest that increased catabolism of lipids induced by EPA\&DHA in the small intestine contributes to the complex and beneficial effects of n-3 PUFA of 

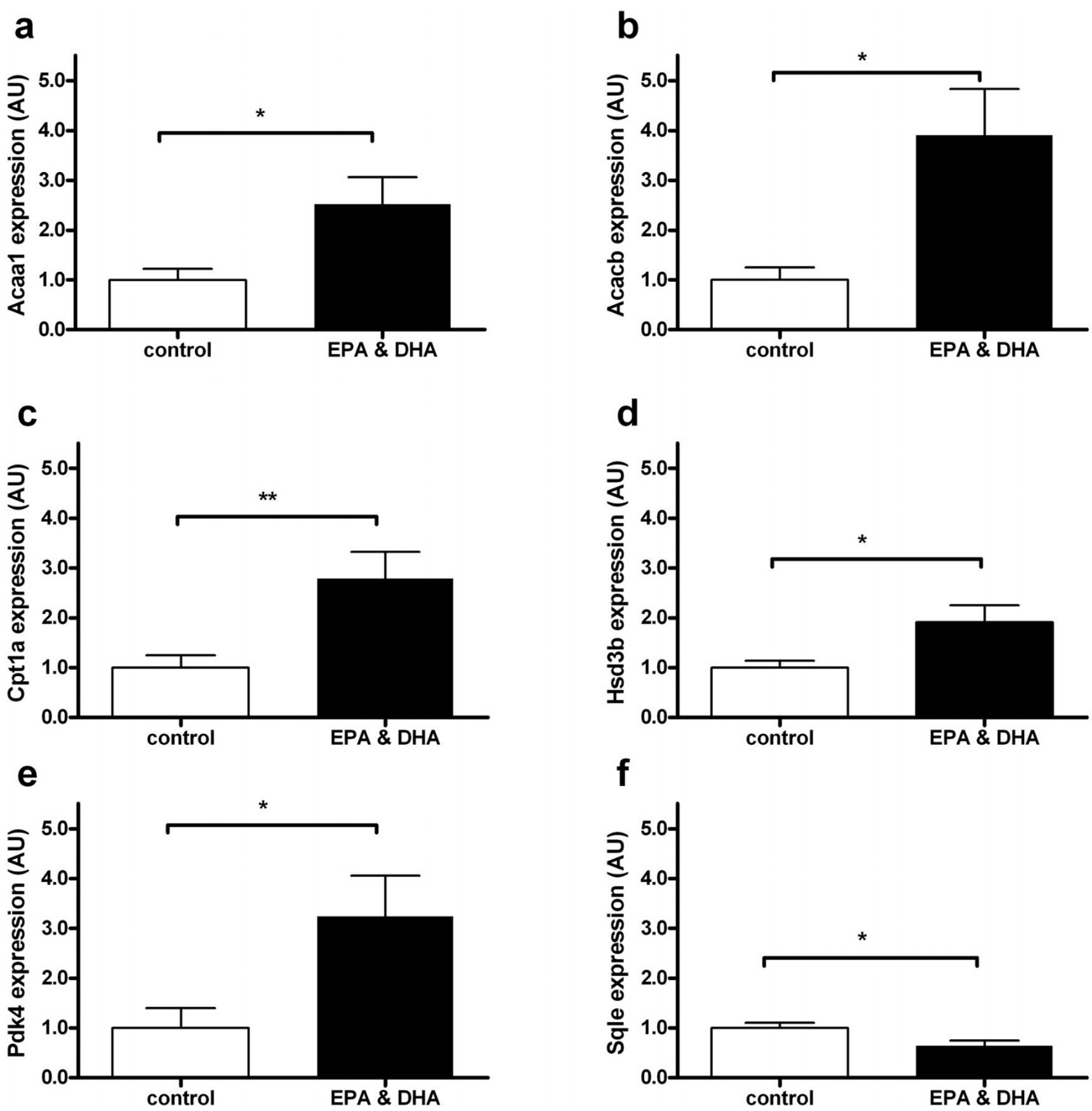

\section{Figure I}

Gene expression in small intestine. Quantatative real-time PCR ( $q R T-P C R$ ) was used to determine normalized gene expression levels in small intestine of individual wildtype mice that received the control diet $(n=10$, white bars) or EPA\&DHA diet ( $n=1$ I, black bars). Gene expression levels were normalized using calnexin and averaged per group; the mean expression level of the control group was arbitrarily set at I. Bars are presented as mean \pm standard error. Genes shown (with the mean ratio between the groups shown in parenthesis) are a Acaal (2.52), b Acacb (3.9l), c Cptla (2.79), d Hsd3b (I.9l), e Pdk4 (3.24), f Sqle (-I.55). Statistical significance was analyzed using Student's t-test: $* p<0.05$, ** $p<0.0$ I. AU, arbitrary units.

marine origin. The small intestine mediates the entry of nutritional lipids and is one of the main sites of $\beta$-oxidation [32-34]. Therefore, an increase in lipid oxidation in the intestine may exert a hypolipidemic effect, i.e. one of the most pronounced effects of EPA and DHA in mammals (reviewed in $[1,3]$ ). This effect of EPA\&DHA in the 

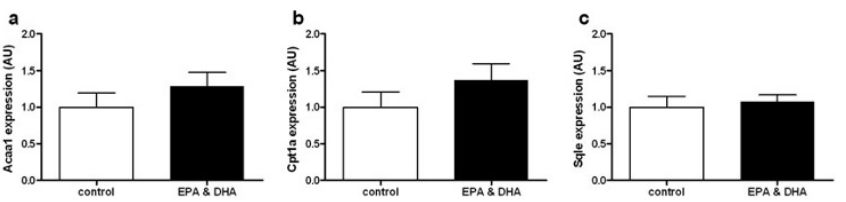

Figure 2

Gene expression in colon. qRT-PCR was used to determine normalized gene expression levels in colon of individual wildtype mice that received the control diet $(n=12$, white bars) or EPA\&DHA diet ( $n=1$, black bars). Gene expression levels were normalized using calnexin and averaged per group; the mean expression level of the control group was arbitrarily set at I. Bars are presented as mean \pm standard error. Genes and fold changes in parenthesis shown are a Acaal (1.29), b Cptla (I.37), c Sqle (1.08). All changes are non-significant $(p>0.2$, Student's t-test). AU, arbitrary units.

intestine is surprisingly similar to the enhanced lipid oxidation induced by diacylglycerols versus triacylglycerols $[18,19]$. These two types of treatments both have relatively little effects in liver and muscle ([20]; own unpublished data). In contrast, in white adipose tissue, intake of EPA\&DHA induced genes of fatty acid oxidation, as well as quite specifically, mitochondrial biogenesis [8]. Taken together, EPA and DHA orchestrate gene expression adaptations in many tissues, including the intestine.
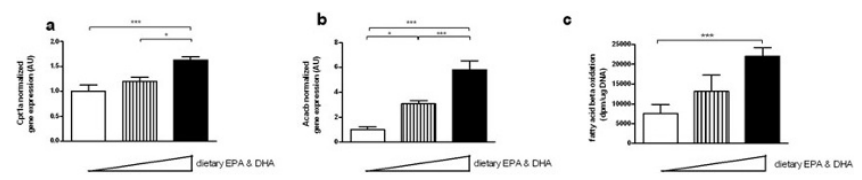

\section{Figure 3}

Dose-dependent intestinal gene expression and fatty acid beta oxidation. In a second animal experiment, intestinal gene expression analysis was used to confirm and biologically validate observations in a dose-dependent manner. Gene expression analysis was performed using QRT-PCR of small intestinal samples of individual wildtype mice that received the control diet ( $n=9$, white bars), EPA\&DHA diet ( $n=9$, black bars), and intermediate levels of dietary EPA\&DHA ( $n=9$, hatched bars). The triangle shows increasing dietary EPA\&DHA content. Gene expression levels were normalized using calnexin and averaged per group; the mean expression level of the control group was arbitrarily set at I. Bars represent mean \pm standard error. Genes shown are a: Cptla, and b: Acacb. Functional fatty acid beta oxidation was used for biological validation (c). Intestinal tissue fatty acid beta oxidation ( $n=7-9$ ) is shown for mice that received control diet (white bars), EPA\&DHA diet (black bars), and intermediate levels of dietary EPA-DHA (hatched bars). The triangle shows increasing dietary EPA\&DHA content. Statistical significance was analyzed using one-way ANOVA and Tuckey's post hoc tests: ${ }^{*} \mathrm{p}<0.05$, *** $\mathrm{p}<0.0 \mathrm{I}$. AU, arbitrary units.
Recent studies addressing the gene expression changes of intestinal tissue upon fish oil or fatty acids, focussed on barrier genes only [16] or a focussed limited number of genes by qRT-PCR [34], while here a whole genome approach was used. This allows for detection of changes not only in the most likely pathways, but also in pathways not foreseen. Our study supports the findings by Mori et al. [34] for the majority of their selected genes analyzed (Cpt1a, Mod1, Pdk4, Hmgcs2, Cyp4a10, and Acadm), as well as for barrier gene expression ([16], data not shown). Unexpectedly, we observed intestinal downregulation of cholesterol biosynthesis due to EPA\&DHA, although this is in agreement with a similar effect observed in murine livers after tuna fish oil feeding [11]. In addition, this might coincide with a possible increase in cholesterol absorption as observed from ScarB1, Cd36, Abca1 and Ela3B gene expression, even with identical cholesterol content of the diets. Intracellular homeostasis may decrease cholesterol biosynthesis to counteract increased influx. Maintenance of homeostasis is supported by non differential expression of Soat2/ACAT, involved in cholesterol esterification and of other genes in cholesterol metabolism (HMG-CoA reductase, Npc1l1, Mttp, Abcg5, Abcg8, Nr1h2 (LXR) and Nr1h3 (LXR)). The intestinal lack of regulation of Srebf1/SREBP further strengthen the observations that PUFA regulation of SREBP that accounts for PUFA-mediated suppression of gene expression seems to be liver-specific [13]. Furthermore, of the regulatory machine known to be induced by DHA and EPA (PPARs, LXRs, HNF4A, and SREBPs), only PPAR $\alpha$ showed differential expression in murine small intestine. This is further supported by our promoter-analysis of the differentially regulated genes, which showed PPAR $\alpha$ as the major transcription factor involved.

Moreover, most if not all tissues analysed thus far show an increased energy metabolism upon n-3 FFA, and our results support the notion of the beneficial effects of fish oils independent of its n-3 effect.

Genes engaged in lipid oxidation and ketogenesis are in general upregulated in small intestine [35], liver [20], and skeletal muscle [36] by an increase in dietary fat content. When activated in the muscle, ketogenesis marks a metabolic disconnection between $\beta$-oxidation and tricarboxylic acid cycle and could lead to insulin resistance [36]. In our study however, we compared diets with equal fat content in control and intervention groups, which only differed in their fatty acid composition. This implies that marine PUFAs specifically induce lipid catabolism in intestine. Furthermore, in comparison with another high fat Western diet [35], we observed similar (up or down) gene expression regulation by EPA\&DHA diet (e.g. Angplt4, Gsn, and Smpdl3), as well as an inverse regulation (e.g. ApoC2 and H2Q10 are upregulated by a high-fat diet 
[35], but downregulated by EPA\&DHA). Differences might be explained by the fatty acid content in the diets used.

Despite decreased adiposity due to EPA\&DHA in the diet, these fatty acids have not affected total energy intake $[8,22]$, and also content of lipids in faeces was unaffected [22]. This strongly suggests a higher energy expenditure in the animals exposed to EPA\&DHA. The results presented here indicate that one of the organs that physiologically contribute to increased oxidation of fatty acids is the small intestine.

\section{Conclusion}

In conclusion, we present data showing the involvement of small intestine in the complex changes of lipid metabolism exerted by long term dietary intake of EPA and DHA by gene expression analysis and functional ex-vivo beta oxidation analysis. Furthermore, we show that these effects are regulated in a dose-dependent manner. In view of its large contribution to overall energy metabolism, modulation of gene expression and metabolism in the intestine by dietary lipids, and especially long-chain n-3 PUFA of marine origin, represents a promising target for the prevention of obesity and associated co-morbidities.

\section{Methods}

\section{Animals and diets}

In the first experiment, male 4-month-old C57BL/6J mice were maintained for 4 weeks on semisynthetic high-fat (20\% wt/wt) diets differing in the composition of $n-3$ PUFA. These mice were already used in our previous study [8]. Two isocaloric diets $[8,22]$ were used $(n=12)$ : control sHFf diet which contained flax-seed oil (rich in ALA) as the only lipid source, or the sHFf-F2 diet, which had the same composition except that $44 \%$ of the lipids were replaced by a n-3 PUFA concentrate containing 6\% EPA and 51\% DHA (EPAX 1050TG; EPAX AS, Lysaker, Norway). This diet is denoted as EPA\&DHA throughout the study. At the end of the experiment, mice were killed by cervical dislocation and small intestine from $3 \mathrm{~cm}$ under the stomach to caecum was isolated and cut lengthwise. The intestine was washed in $154 \mathrm{mM} \mathrm{KCl}$ and scraped. The epithelial cells were collected, frozen in liquid nitrogen and stored at $-80^{\circ} \mathrm{C}$. This procedure was also performed for $5 \mathrm{~cm}$ of colon.

In the second experiment, subgroups $(\mathrm{n}=9)$ of male 4month-old $\mathrm{C} 57 \mathrm{BL} / 6 \mathrm{~J}$ mice were fed either (i) control obesity-promoting high-fat (35\% lipids wt/wt; cHF) diet derived from standard chow diet based on corn oil, or (ii) and (iii) cHF diet with 15 and $44 \%$ of lipids, respectively, replaced by EPAX 1050TG (cHF-F1 and cHF-F2, respectively; see [22]). After 6 weeks, intestinal tissue was isolated for fatty acid beta oxidation and gene expression analysis by quantitative RT-PCR (qRT-PCR) only. Macronutrient composition, energy density, and fatty acid composition of dietary lipids of all the diets used in this study is well characterised [22]. The experiments were conducted under the guidelines for the use and care of laboratory animals of the Institute of Physiology, Academy of Sciences of the Czech Republic.

\section{RNA isolation}

Extraction of total RNA was performed with the use of TRIzol (Invitrogen, Breda, The Netherlands). RNeasy columns (Qiagen, Venlo, The Netherlands) were used to purify the RNA. Quantitative and qualitative measures were performed using Nanodrop ND-1000 Spectrophotometer (NanoDrop Technologies, Delaware, USA). For the first animal experiment, four samples did not pass quality thresholds and were excluded from further analyses (two control small intestine samples and one EPA\&DHA small intestine sample and one EPA\&DHA colon sample). The remaining RNA samples were pooled per tissue per diet and integrity was analyzed after keeping the samples for 1 hour at $37^{\circ} \mathrm{C}$ using an Agilent bioanalyzer (Agilent Technologies, Amstelveen, The Netherlands) with a RNA 6000 Nano LabChip kit, according to the manufacturer's instructions. The two pooled colon samples were not used further due to their apparent partial degradation (RNA Integrity Number $(\mathrm{RIN})<7.0)$. All individual RNA samples from the second animal experiment passed quality control criteria, and were used for subsequent qRT-PCR analysis.

\section{Array hybridization and scanning}

The pools of small intestine RNA samples (control ( $\mathrm{n}=$ $10)$ and EPA\&DHA $(\mathrm{n}=11)$ ) were hybridized on separate Affymetrix MOE430_2 GeneChip mouse arrays (Santa Clara, CA, USA). This array contains 45,102 probesets, detecting over 39,000 transcripts that represent 16,579 unique genes. Detailed methods for labelling and subsequent hybridizations to the arrays are described in the eukaryotic section in the GeneChip Expression Analysis Technical Manual Rev. 3 from Affymetrix, and are available upon request. Arrays were scanned on a GeneChip Scanner 3000 (Affymetrix).

\section{Microarray data analysis}

Quality of the data was assessed on diagnostic plots generated from the raw, non-processed data, as described [37]. All arrays passed these strict criteria and were included in the analyses.

The Affymetrix default algorithm (MAS 5.0) was used to summarize data and significance of observed gene expression changes (present/absent call per probeset per array, fold change (FC) between normalized arrays, and its significance by the p-value). In total, 24270 probesets (54\%) 
showed expression at least in either one of the arrays. Significant differentially expressed probesets were identified by direct comparison between the two dietary groups for all probesets called present on both arrays. Probesets that satisfied the general Affymetrix criterion of t-test probability $<0.27 \%$ ( $p$-value $<0.0027$ ) were considered to be significantly regulated, and these were further investigated. Probesets were annotated using information provided by Affymetrix (release of July 12th, 2006) and all gene symbols are presented throughout the article according to Mouse Genome Informatics [38].

Pathway analysis, including ranking, was done using Metacore (GeneGo, St. Joseph, MI, USA). The data of functionally annotated genes only were analyzed in two subsets. In order to successfully rank the most prominent differentially regulated pathway(s), a large set of genes was analyzed. In this way, those pathways showing a high number of significant regulated genes, given the (high) number of genes expressed within the pathway, will rank highest. The first subset comprised those genes of which expression was present in both dietary conditions. Pathway analysis was done using a cut-off of absolute FC $\geq 1.5$ or $\geq 2.0$ and the p-value per gene as provided by MAS5.0, in order to increase detection of biological relevant regulated pathways. The second subset comprised probesets expressed in only one dietary group, and therefore absent in the other ( $F C \geq 2.0)$. The given FCs as generated by MAS 5.0 were used, although in view of the absence of expression under one dietary condition these may not be reliable, hence the choice of separate analysis. Array data have been submitted to the Gene Expression Omnibus, accession number GSE11936.

\section{Promoter analysis to identify transcription factor regulation}

All differentially regulated probesets, as shown in Table 1, were analyzed using Genomatix BiblioSphere Pathway Edition software, without any pre-selection in up- or down-regulation. Unique genes were identified and those genes plus gene-gene interactions were filtered [39] for organ-specificity using MeSH [Medical Subject Heading] "Digestive System" [A03]. This resulted in a set of 50 unique input genes. Promoter regions of around $650 \mathrm{bp}$ upstream of transcription start site per gene were analysed for known transcription factor binding sites (TFBS) combined with prior knowledge in literature. Criteria for this analysis were: only direct interactions were considered, a minimum of 3 published articles should describe the functional interaction (so called "function word level B2"), and for inclusion of a transcription factor (TF) showing TF-gene interaction, the additional criterion of at least two different input genes having this specific TFBS was compulsory. In this way we analyzed specifically only TF-gene interactions relevant for the gastrointestinal tract.

\section{Quantitative real time PCR}

qRT-PCR was performed according to Van Schothorst et al. [40]. Briefly, $1 \mu \mathrm{g}$ of total RNA was used for cDNA synthesis. Primers were designed using Beacon Designer (Biosoft International, Palo Alto, USA) and ordered by Biolegio (Malden, The Netherlands). Gene symbols, names, accession numbers and primer sequences are listed in an additional file [see Additional file 5]. Analysis of the reaction efficiency [41] was performed with a dilution series of pooled cDNAs serving as standard curve. Briefly, after a 3 minute denaturation at $94^{\circ} \mathrm{C}, 40$ cycles of 15 seconds at $94^{\circ} \mathrm{C}$ and 30 seconds at $59^{\circ} \mathrm{C}$, were followed by a melting curve gradient. Calnexin (Canx) and hypoxanthine guanine phosphoribosyl transferase 1 (Hprt1) were used as reference genes based on least variation observed in microarray data analysis. Canx showed most stable expression and was used and shown in all cases. Endorsable data were obtained using Hprt1. $\mathrm{C}_{\mathrm{t}}$-values of the two pools of dietary groups were measured for 17 genes in triplo and averaged, followed by calculation of the relative gene expression using the $2^{-\Delta \Delta \mathrm{CT}}$ method [42].

Relative target gene expression was measured in more detail using individual samples for the genes Cpt1a, Acaa1a, Sqle, Acacb, Pdk4 and Hsd3b, of which the latter three were not analysed using pooled RNA samples. Samples were run in duplicate, averaged, and relative gene expression was calculated using the standard curve method. qRT-PCR analyses for the second animal experiment were performed using individual RNA samples for target genes Cpt1 $a$ and $A c a c b$, as described above.

All values are presented as mean \pm SE. In case of only 2 groups (first experiment), differences between groups were analyzed using Student t-tests with two-tailed, unequal variances and significance expressed at $\mathrm{p}<0.05$, or lower as indicated, while differences between three groups (second experiment) were analysed using one-way ANOVA and Tuckey's posthoc tests and significance expressed at $\mathrm{p}<0.05$, or lower as indicated.

\section{Fatty acid beta oxidation}

Intestinal fatty acid beta oxidation was measured as published [43]. Briefly, samples were incubated in KrebsRinger bicarbonate buffer with $\left[{ }^{14} \mathrm{C}(\mathrm{U})\right]$-palmitate, ${ }^{14} \mathrm{CO}_{2}$ was trapped in hyamine hydroxide, quantified by liquid scintillation counting, and oxidation rate was normalized to tissue DNA content [44]. All data are presented as mean \pm SE. Differences between groups were analyzed using one-way ANOVA and Tuckey's post hoc tests and considered statistically significance at $\mathrm{p}<0.05$.

\section{Authors' contributions}

EvS, PF, NFvH, JKo and JKe conceived the studies, supervised the execution and drafted the mansucript. EvS, PF, 
$\mathrm{OK}, \mathrm{AB}, \mathrm{JM}, \mathrm{CV}$, and $\mathrm{GH}$ performed animal studies, RNA isolation, qRT-PCRs, microarray hybridisation and analyses, or fatty acid beta oxidation measurements. All authors have read and approved the final manuscript.

\section{Additional material}

\section{Additional file 1}

Differentially regulated probesets expressed in both dietary groups. The data provided represent the statistical significant differentially expressed probesets of the microarrays which are shared between the two dietary groups (Fold change 2.0).

Click here for file

[http://www.biomedcentral.com/content/supplementary/14712164-10-110-S1.pdf]

\section{Additional file 2}

Additional data analysis of gene expression differences. Additional and extended microarray data analysis results are described in more detail. Click here for file

[http://www.biomedcentral.com/content/supplementary/14712164-10-110-S2.pdf]

\section{Additional file 3}

Transcription factors enriched for involvement with differentially expressed genes. The data provided represent identified transcription factors on the basis of an enriched presence of TFBS in the promoter region of the set of significantly differentially regulated genes, combined with data from scientific publications.

Click here for file

[http://www.biomedcentral.com/content/supplementary/14712164-10-110-S3.pdf]

\section{Additional file 4}

Differentially regulated probesets expressed in only one dietary group. The data provided represent all differentially expressed probesets of the microarrays which are unique for either one of the dietary groups. Click here for file

[http://www.biomedcentral.com/content/supplementary/14712164-10-110-S4.pdf]

\section{Additional file 5}

Real-time quantitative PCR: genes and primers. Gene Symbols, Names, Annotation (RefSeq), and primer sequences for genes analyzed using $q R T$-PCR are shown.

Click here for file

[http://www.biomedcentral.com/content/supplementary/14712164-10-110-S5.pdf]

\section{Acknowledgements}

This work was supported by the Dutch Ministry of Economic Affairs through the Innovation Oriented Research Program on Genomics, IOP Genomics IGEOI016, the Dutch Ministry of Agriculture, Nature management and food quality (8037I7390 I; EvS, NFvH, AB, JM, CV, GH, and JKe), by the Czech Science Foundation (IM6837805002; PF, OK and JKo), and by EPAX AS, Lysaker, Norway. The research is performed in the context of MITOFOOD.

\section{References}

I. Lombardo YB, Chicco AG: Effects of dietary polyunsaturated n3 fatty acids on dyslipidemia and insulin resistance in rodents and humans. A review. J Nutr Biochem 2006, I 7(I): I-I3.

2. Mills SC, Windsor AC, Knight SC: The potential interactions between polyunsaturated fatty acids and colonic inflammatory processes. Clin Exp Immunol 2005, I 42(2):2 I 6-228.

3. Sampath $\mathrm{H}, \mathrm{Ntambi} \mathrm{M}$ : Polyunsaturated fatty acid regulation of genes of lipid metabolism. Annu Rev Nutr 2005, 25:3 I 7-340.

4. Geleijnse JM, Brouwer IA, Feskens EJ: Risks and benefits of omega 3 fats: health benefits of omega 3 fats are in doubt. BM/2006, 332(7546): $915-916$.

5. Hooper L, Thompson RL, Harrison RA, Summerbell CD, Ness AR, Moore HJ, Worthington HV, Durrington PN, Higgins JP, Capps NE, et al.: Risks and benefits of omega 3 fats for mortality, cardiovascular disease, and cancer: systematic review. BM] 2006, 332(7544):752-760.

6. Buettner R, Parhofer KG, Woenckhaus M, Wrede CE, KunzSchughart LA, Scholmerich J, Bollheimer LC: Defining high-fat-diet rat models: metabolic and molecular effects of different fat types. J Mol Endocrinol 2006, 36(3):485-50I.

7. Clarke SD, Jump D: Polyunsaturated fatty acids regulate lipogenic and peroxisomal gene expression by independent mechanisms. Prostaglandins Leukot Essent Fatty Acids 1997, 57(I):65-69.

8. Flachs P, Horakova O, Brauner P, Rossmeisl M, Pecina P, Franssen-van Hal N, Ruzickova J, Sponarova J, Drahota Z, Vlcek C, et al.: Polyunsaturated fatty acids of marine origin upregulate mitochondrial biogenesis and induce beta-oxidation in white fat. Diabetologia 2005, 48( I I ):2365-2375.

9. Ide T: Interaction of fish oil and conjugated linoleic acid in affecting hepatic activity of lipogenic enzymes and gene expression in liver and adipose tissue. Diabetes 2005, 54(2):4I2-423.

10. Jump DB, Botolin D, Wang Y, Xu J, Christian B, Demeure O: Fatty acid regulation of hepatic gene transcription. J Nutr 2005, I35( I I):2503-2506.

II. Kim HJ, Takahashi M, Ezaki O: Fish oil feeding decreases mature sterol regulatory element-binding protein I (SREBP-I) by down-regulation of SREBP-I $c$ MRNA in mouse liver. A possible mechanism for down-regulation of lipogenic enzyme mRNAs. J Biol Chem 1999, 274(36):25892-25898.

12. Kitajka Kr, Sinclair AJ, Weisinger RS, Weisinger HS, Mathai M, Jayasooriya AP, Halver JE, Puskás LG: Effects of dietary omega-3 polyunsaturated fatty acids on brain gene expression. Proc Natl Acad Sci USA 2004, I 0 I(30): I093।-10936.

13. Ntambi J, Bené $\mathrm{H}$ : Polyunsaturated fatty acid regulation of gene expression. J Mol Neurosci 200I, I 6(2-3):273-278.

14. Sampath $\mathrm{H}, \mathrm{Ntambi} \mathrm{JM}$ : Polyunsaturated fatty acid regulation of gene expression. Nutr Rev 2004, 62(9):333-339.

15. Sekiya M, Yahagi N, Matsuzaka T, Najima Y, Nakakuki M, Nagai R, Ishibashi S, Osuga J, Yamada N, Shimano H: Polyunsaturated fatty acids ameliorate hepatic steatosis in obese mice by SREBPI suppression. Hepatology 2003, 38(6): | 529- I539.

16. de Vogel-van den Bosch HM, Bunger M, de Groot PJ, Bosch-Vermeulen H, Hooiveld GJ, Muller M: PPARalpha-mediated effects of dietary lipids on intestinal barrier gene expression. $B M C$ Genomics 2008, 9(I):23I.

17. Krebs HA: Body size and tissue respiration. Biochim Biophys Acta 1950, 4( I-3):249-269.

18. Murase T, Aoki M, Wakisaka T, Hase T, Tokimitsu I: Anti-obesity effect of dietary diacylglycerol in C57BL/6J mice: dietary diacylglycerol stimulates intestinal lipid metabolism. J Lipid Res 2002, 43(8): 1312-1319.

19. Murase T, Nagasawa A, Suzuki J, Wakisaka T, Hase T, Tokimitsu I: Dietary alpha-linolenic acid-rich diacylglycerols reduce body weight gain accompanying the stimulation of intestinal betaoxidation and related gene expressions in C57BL/KsJ-db/db mice. J Nutr 2002, I 32(10):3018-3022.

20. Kondo H, Minegishi Y, Komine Y, Mori T, Matsumoto I, Abe K, Tokimitsu I, Hase T, Murase T: Differential regulation of intestinal lipid metabolism-related genes in obesity-resistant $A / J$ vs. obesity-prone C57BL/6J mice. Am J Physiol Endocrinol Metab 2006, 29 I(5):EI092-EI099.

21. Ikemoto S, Takahashi M, Tsunoda N, Maruyama K, Itakura H, Ezaki O: High-fat diet-induced hyperglycemia and obesity in mice: dif- 
ferential effects of dietary oils. Metabolism 1996, 45(I2): I539-1546.

22. Ruzickova J, Rossmeisl M, Prazak T, Flachs P, Sponarova J, Veck M, Trrzicka E, Bryhn M, Kopecky J: Omega-3 PUFA of marine origin limit diet-induced obesity in mice by reducing cellularity of adipose tissue. Lipids 2004, 39( I 2): I I77- I I 85.

23. Flachs P, Mohamed-Ali V, Horakova O, Rossmeisl M, HosseinzadehAttar MJ, Hensler M, Ruzickova J, Kopecky J: Polyunsaturated fatty acids of marine origin induce adiponectin in mice fed a highfat diet. Diabetologia 2006, 49(2):394-397.

24. Afman L, Muller M: Nutrigenomics: from molecular nutrition to prevention of disease. J Am Diet Assoc 2006, I06(4):569-576.

25. Pagmantidis V, Meplan C, van Schothorst EM, Keijer J, Hesketh JE: Supplementation of healthy volunteers with nutritionally relevant amounts of selenium increases the expression of lymphocyte protein biosynthesis genes. Am J Clin Nutr 2008, 87(I): $181-189$.

26. Sugden MC: PDK4: A factor in fatness? Obes Res 2003, II(2):167-169.

27. Mandard S, Muller M, Kersten S: Peroxisome proliferator-activated receptor alpha target genes. Cell Mol Life Sci 2004, 6I(4):393-4I6.

28. Bunger M, Bosch HM van den, Meijde I van der, Kersten S, Hooiveld G], Muller M: Genome-wide analysis of PPARalpha activation in murine small intestine. Physiol Genomics 2007, 30(2): I92-204.

29. Hui DY, Labonte ED, Howles PN: Development and physiological regulation of intestinal lipid absorption. III. Intestinal transporters and cholesterol absorption. Am J Physiol Gastrointest Liver Physiol 2008, 294(4):G839-843.

30. Kersten S: Peroxisome proliferator activated receptors and lipoprotein metabolism. PPAR Res 2008, 2008: 132960.

31. Calder PC: Polyunsaturated fatty acids, inflammatory processes and inflammatory bowel diseases. Mol Nutr Food Res 2008 52(8):885-897.

32. Kelly DP, Gordon Jl, Alpers R, Strauss AW: The tissue-specific expression and developmental regulation of two nuclear genes encoding rat mitochondrial proteins. Medium chain acyl-CoA dehydrogenase and mitochondrial malate dehydrogenase. J Biol Chem 1989, 264(32): |892|-|8925.

33. Nemali MR, Usuda N, Reddy MK, Oyasu K, Hashimoto T, Osumi T, Rao MS, Reddy JK: Comparison of constitutive and inducible levels of expression of peroxisomal beta-oxidation and catalase genes in liver and extrahepatic tissues of rat. Cancer Res 1988, 48(18):5316-5324.

34. Mori T, Kondo H, Hase T, Tokimitsu I, Murase T: Dietary fish oil upregulates intestinal lipid metabolism and reduces body weight gain in C57BL/6] mice. J Nutr 2007, I37( ( 2):2629-2634

35. de Wit NJ, Bosch-Vermeulen H, de Groot PJ, Hooiveld G], Grootte Bromhaar MM, Jansen J, Muller M, Meer R van der: The role of the small intestine in the development of dietary fat-induced obesity and insulin resistance in C57BL/6J mice. BMC Med Genomics 2008, I(I): I4

36. Muoio DM, Newgard CB: Obesity-Related Derangements in Metabolic Regulation. Annu Rev Biochem 2006, 75:367-40I.

37. Bolstad BM, Collin F, Brettschneider J, Simpson K, Cope L, Irizarry RA, Speed TP, Gentleman RC, Huber W, Irizarry R, et al: Quality Assessment of Affymetrix GeneChip Data. In Bioinformatics and Computational Biology Solutions Using $R$ and Bioconductor New York Springer; 2005:33-47.

38. The Mouse Genome Informatics Database [http://www.infor matics.jax.org/]

39. Seifert M, Scherf M, Epple A, Werner T: Multievidence microarray mining. Trends Genet 2005, 21(10):553-558.

40. Van Schothorst EM, Keijer J, Pennings JLA, Opperhuizen A, Brom CE Van den, Kohl T, Franssen-vanHal NLM, Hoebee B: Adipose gene expression response in lean and obese mice to short term dietary restriction. Obesity 2006, I 4(6):974-979.

41. Rasmussen R, Meuer S, Wittwer C, Nakagawara K: Quantification on the LightCycler. In Rapid cycle real-time PCR, methods and applications Heidelberg: Springer Press; 2001:21-34.

42. Livak KJ, Schmittgen TD: Analysis of relative gene expression data using real-time quantitative PCR and the 2(-Delta Delta C(T)) Method. Methods 200I, 25(4):402-408.

43. Kus V, Prazak T, Brauner P, Hensler M, Kuda O, Flachs P, Janovska P, Medrikova D, Rossmeisl M, jilkova Z, et al.: Induction of muscle thermogenesis by high-fat diet in mice: association with obesity-resistance. Am 」 Physiol Endocrinol Metab 2008, 295(2):E356-367.

44. Stefl B, Janovska A, Hodny Z, Rossmeisl M, Horakova M, Syrovy I, Bemova J, Bendlova B, Kopecky J: Brown fat is essential for coldinduced thermogenesis but not for obesity resistance in aP2Ucp mice. Am J Physiol 1998, 274(3 Pt I):E527-533.
Publish with Bio Med Central and every scientist can read your work free of charge

"BioMed Central will be the most significant development for disseminating the results of biomedical research in our lifetime. "

Sir Paul Nurse, Cancer Research UK

Your research papers will be:

- available free of charge to the entire biomedical community

- peer reviewed and published immediately upon acceptance

- cited in PubMed and archived on PubMed Central

- yours - you keep the copyright 